九州大学学術情報リポジトリ

Kyushu University Institutional Repository

\title{
Silphid Fauna of Chejudo Island, Korea
}

Nomura, Shuhe i

Lee, Chang Eon

https://doi.org/10.5109/2558

出版情報 : ESAKIA. 32, pp.81-86，1992-03-31. Entomological Laboratory，Faculty of Agriculture， Kyushu University

バージョン :

権利関係 : 


\title{
Silphid Fauna of Chejudo Island, Korea ${ }^{1), 2)}$
}

\author{
Shûhei NOMURA \\ Entomologica Laboratory, Faculty of Agriculture, Kyushu University, \\ Fukuoka 812, Japan \\ and \\ Chang Eon LEE \\ Department of Biology, College of Natural Sciences, Kyungpook National University, \\ Taegu, 702-701, Korea
}

\begin{abstract}
.
The silphids known from Chejudo Island, ten in all are reviewed and Eusilpha bicolor is reported for the first time.
\end{abstract}

\section{Introduction}

The silphid fauna of Chejudo Island is well-known since Okamoto (1924). He reported 6 species from Chejudo for the first time. Cho (1957) reviewed Korean Coleoptera and listed 21 silphid species without comment on Cheju fauna. In 1963 he recorded 8 species from this island, in which two species Nicrophorus concolor and Ptomascopus morio were newly added.' Kim (1978) presented the distributions of 15 Korean species with new distribution record of Eusilpha brunneus from Cheju. Lee, Kim \& Kim (1985) added Nicrophorus quadripunctatus to the fauna of this island. Paik (1985) revised the Korean Silphidae and Catopidae with a key to 23 silphid species.

This short report presents a check-list of the silphid fauna of Chejudo from the Korean literature with a new record of Eusilpha bicolor on the basis of our own collection.

\section{Family SILPHIIDAE}

\section{Tribe Nicrophorini}

\section{Nicrophorus japonicus Harold}

1) Results from the Korea-Japan Co-operative Science Program on "The Evolution and Biogeography of the Insects in the East Asia". No. 7.

2) Contribution from the Entomological Laboratory, Faculty of Agriculture. Kyushu University, Fukuoka (Ser. 4, No. 41) 
Necrophorus japonicus Harold, 1877, Deutsch. Ent. Zeitschr., 21: 345; Cho, 1957, Hum. Sci., Korea Univ., 2: 32 .

Necrophorus japonica: Okamoto, 1924, Bull Agric. Expt. Stat. Gov. -Gen. Chosen, 1: 168.

Nicrophorus japonicus: Cho, 1963, Hum. Sci. Korea Univ., 6: 46; Seok, 1970, Ins. Faun. Is. Querpart: 71; Kim, 1978, Dist. Atlas Ins. Korea, Col., 2: 64; Lee, Kim \& Kim, 1985, Rep. acad. Surv. Hallasan (Mt.) Nat. Pres.: 403; Paik, 1985, Corentomon, Suwon, 1(1): 3.

Distribution. Korea, China, Mongolia, Formosa, Japan.

\section{Nicrophorus concalor Kraatz}

(Fig. A)

Necrophorus concolor Kraatz, 1877, Deutsch. ent. Zeitschr., 21: 100; Cho, 1957, Hum. Sci. Korea Univ., 2: 33.

Nicrophorus concolor: Cho, 1963, Hum. Sci. Korea Univ., 6: 46; Lee, Kim \& Kim 1985, Rep. acad. Surv. Hallasan (Mt.) Nat. Pres.: 403; Paik, 1985, Corentomon, Suwon, 1(1): 4.

Nicrophorus concoler : Kim, 1978, Dist. Atlas Ins. Korea, 2: 61 (misspelling).

Specimens examined: 2 males, Yongshil, Mt. Hallasan, 27. vii. 1990, S. Nomura leg.

Distribution. Korea, China, Formosa, Japan.

\section{Nicrophorus quadripunctatus Kraatz}

(Fig. B)

Necrophorus quadripunctatus Kraatz, 1877, Deutsch. ent. Zeitschr., 21: 101.

Nicrophorus quadripunctatus : Kim, 1978, Col., Diit. Atlas Ins. Korea, 2: 63; Lee, Kim \& Kim, 1985, Rep. acad. Surv. Hallasan (Mt.) Nat. Pres.: 403; Paik, 1985, Corentomon, Suwon, 1(1): 3.

Specimens examined: 1 male 1 female, Kaewol Bridge, Mt. Hallasan, 20. v. 1990, S. Nomura leg; 5 males 2 females, Yongshil, Mt. Hallasan, 27. vii. 1990, S. Nomura leg.

Distribution. Korea, China, Japan.

\section{Ptomascopus morio Kraatz}

Ptomaswpus morio Kraatz, 1877, Deutsch. ent. Zeitschr., 21: 104; Cho, 1957, Hum. Sci. Korea Univ., 2: 33; 1963, Hum. Sci. Korea Univ., 6: 47; Kim, 1978, Col., Dist. Atlas Ins. Korea, 2: 62; Lee, Kim \& Kim, 1985, Rep. acad. Surv. Hallasan (Mt.) Nat. Pres.: 403; Paik, 1985, Corentomon, Suwon, 1(1): 4.

Distribution. Korea, China, Formosa, Japan.

\section{Tribe Necrodini}

\section{Necrodes asiaticus Portevin}

(Fig. C)

Necrodes asiaticus Portevin, 1922, Bull. Mus. Hist. Nat., Paris, 1922: 507; 1926, Silph., Encycl. Ent., 6: 162; Kim, 1978, Dist. Atlas Ins. Korea, 2: 59; Paik, 1985, Corentomon, Suwon, 1(1): 3. Necrodes littoralis : Okamoto, 1924 (nec. Linnaeus, 1758), Bull Agric. Expt. Stat. Gov. -Gen. Chosen, 1: 
168.

Specimens examined: 1 male, Cheju Junior College, Cheju City, 19. v. 1990, S. Nomura leg.; 2 males 8 females, Kaewol Bridge, Mt. Hallasan, 23. vii. 1990, S. Nomura leg.; 1 male 2 females, same locality as above, 26. vii. 1990, S. Nomura leg.

Distribution. Korea China, Tibet, Mongolia, Sibelia, India, Japan.

\section{Tribe Silphini}

\section{Thanatophilus sinuatus (Fabricius)}

Silpha sinuatus Fabricius, 1775, Syst. Ent.: 75; Cho, 1957, Hum. Sci. Korea Univ., 2: 31.

Pseudoperta sinuata: Okamoto, 1924, Bull Agric. Expt. Stat. Gov. -Gen. Chosen, 1: 168.

Silpha auripilosa: Cho, 1957 (nec. Portevin, 1926), Hum. Sci. Korea Univ., 2, 1. c.: 31; 1963, Hum. Sci.

Korea Univ., 6: 47; Seok, 1970, Ins. Faun. Is. Querpart: 71; Lee, Kim \& Kim, 1985, Rep. acad.

Sw. Hallasan (Mt.) Nat. Pres.: 403.

Thanatophilus auripilosus : Kim, 1978, Dist. Atlas Ins. Korea, 2: 55.

Thanatophilus sinuatus: Paik, 1985, Corentomon, Suwon, 1(1): 3

Distribution. Korea, China, Siberia, Central Asia, Europe, North Africa, Japan.

\section{Thanatophilus rugasus (Linnaeus)}

Silpha rugosa Linnaeus, 1758, Syst. Nat., 10th ed.: 361.

Pseudopelta rugosa : Okamoto, 1924, Bull Agric. Expt. Stat. Gov. -Gen. Chosen, 1: 169.

Silpha subrugosa : Cho, 1957 (nec. Portevin, 1926), Hum. Sci. Korea Univ., 2: 32; 1963, Hum. Sci. Korea

Univ., 6: 46; Seok, 1970, Ins. Faun. Is. Querpart: 71; Lee, Kim \& Kim, 1985, Rep. acad. Surv.

Hallasan (Mt.) Nat. Pres.: 403.

Thanatophilus subrugosus : Kim, 1978, Dist. Atlas Ins. Korea, 2: 56.

Thanatophilus rugosus : Paik, 1985, Corentomon, Suwon, 1(1): 3

Distribution. Korea, China, Central Asia, Europe, Northern Africa, Siberia, Himaraya, Japan.

\section{Silpha perforata Gebler}

(Fig. D)

Silpha perforata Gebler, 1832, Nouv. Mtm, Mosc.: 41; Cho, 1957, Hum. Sci. Korea Univ., 2: 31; 1963,

Hum. Sci. Korea Univ., 6, 46; Seok, 1970, Ins. Faun. Is. Querpart: 71; Kim, 1978, Dist. Atlas Ins.

Korea, 2: 54; Lee, Kim \& Kim, 1985, Rep. acad. Surv. Hallasan (Mt.) Nat. Pres.: 403; Paik, 1985,

Corentomon, Suwon, 1(1): 3 .

Parasilpha perforata: Okamoto, 1924, Bull. Agric. Expt. Stat. Gov. -Gen. Chosen, 1: 168.

Remarks. This species is divided into five subspecies by Portevin (1926) and the other authors. But we cannot determine the specimen collected from Mt. Hallasan into subspecies because of its bad condition as shown in the figure $\mathrm{D}$.

Specimens d: 1 female, Mt. Hallasan, 25. vii. 1990, S. Nomura leg. 
Distribution. Korea, China, Mongolia, Siberia, Japan (Hokkaido).

\section{Eusilpha (Calosipha) bicolor bicolor (Fairmaire)}

(Fig. E)

Silpha bicolor Fairmaire, 1899. Ann. Soc. ent. Fr., 68: 616; Paik, 1985, Corentomon, Suwon, 1(1): 3.
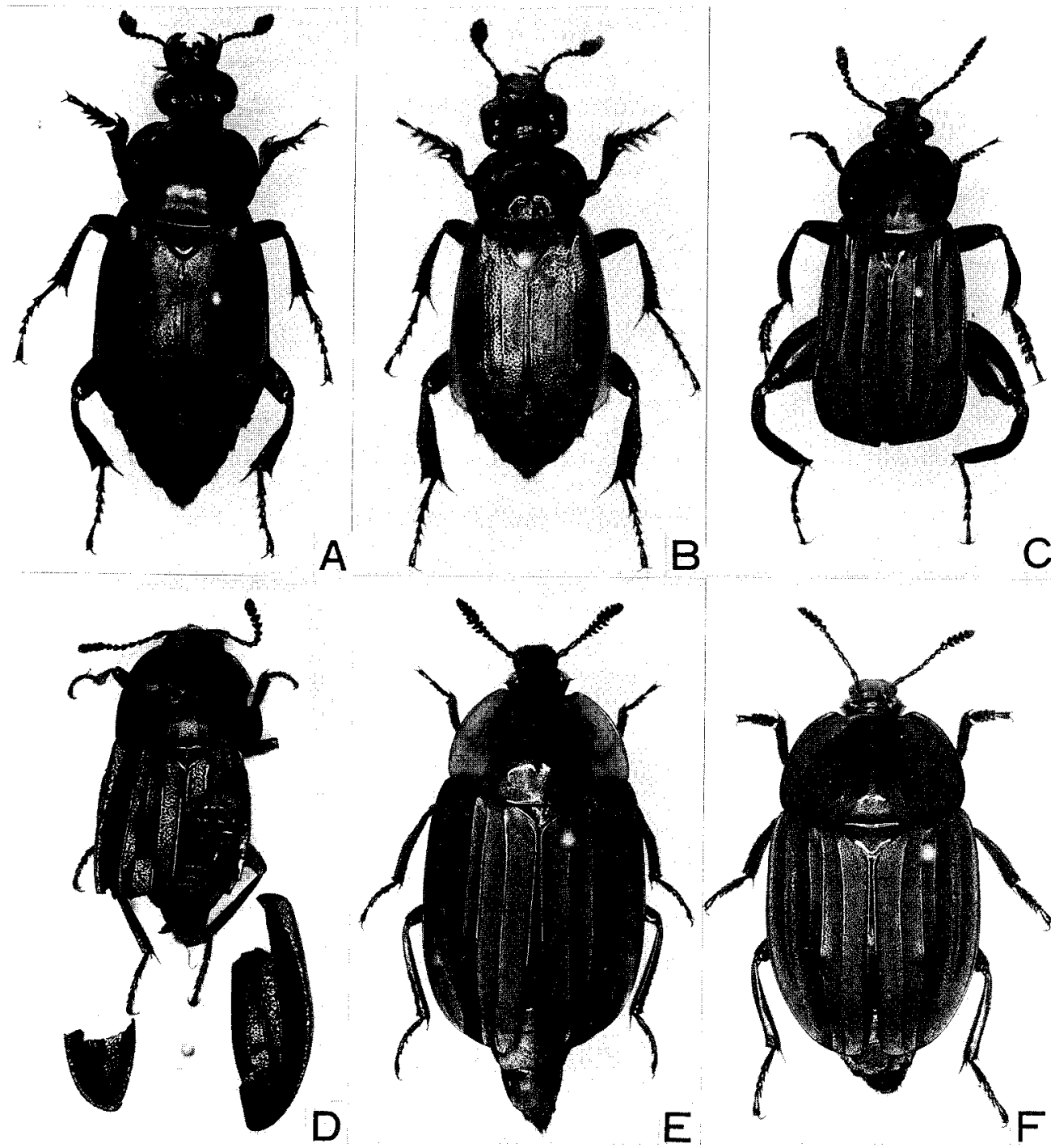

Figures A-F. Dorsal aspects of the silphids collected from Chejudo Is. A, Nicrophorus concolor Kraatz; B, N. quadripunctatus Kraatz; C, Necrodes asiaticus Portevin; D, Silpha perforata Gebler; D, Eusilpha (Calosilpha) bicolor bicolor (Fairmaire); F, E. (E) jacowlewi (Semenow). 
Silpha biwlor bicolor: Niiawa, 1986, Ent. Pap. pres. Kurosawa, Tokyo: 153.

Remarks. This species is recorded from Chejudo Island for the first time. The nominotypicai subspecies known from Korean peninsula is separeted from the subsp. imasakai from Japan by the strongly narrowed pronotum towards apex in both sexes and the less dilated third to fifth antennal segments in the female after Nihikawa (1986).

Specimens examined: 1 female, Kaewol Bridge, Mt.Hallasan, 26. vii. 1990, S. Nomura leg.; 4 females, Yongshil, Mt. Hallasan, 27. vii. 1990, S. Nomura leg.

Distribution. Korea, China, Tibet, Japan.

\section{Eusilpha (Calosilpha) bruneicollis (Kraatz)}

Silpha brunneicollis Kraatz, 1877. Deutsch. ent. Zeitschr.., 21: 106.

Calosilpha brunneicollis: Cho, 1957, Hum. Sci. Korea Univ., 2 (nec, Portevin, 1926): 32; Kim, 1978, Dist. Atlas Ins. Korea, 2; 52.

Eusilpha brunneicollis :; Paik, 1985, Corentomon, Suwon, 1(1): 3.

Distribution. Korea, Formosa?, Japan.

\section{Eusilpha (Eusilpha ) jakowlewi (Semenow)}

(Fig. F)

Silpha jakowlewi Semenow, 1891, Horae, Soc. Ent. Ross. 25: 229; Paik, 1985, Corentomon, Suwon, 1(1): 3; Cho \& Lee, 1986b, Nature and Life, 16 (2): 19.

Sifpha japonica : Cho, 1957, Hum. Sci. Korea Univ., 2 (nec. Motschulsky, 1860) : 203;1963, Hum. Sci. Korea Univ., 6: 46; Lee, Kim\& Kim, 1985, Rep. acad. Surv. Hallasan (Mt.) Nat. Pres.: 403.

Pseudopelta japonica : Okamoto, 1924, Bull Agric. Expt. Stat. Gov. -Gen. Chosen, 1: 169.

Eusilpha japonica : Kim, 1978, Dist. Atlas Ins. Korea, 2: 57.

Specimens examined: 4 males 10 females, Ora-dong, Cheju City, 21. v. 1990, S. Nomura leg.; 1 male, same locality as above, 23. v. 1990, S. Nomura leg.

Distribution. Korea, Mongolia, Japan (Tsushima Is.).

\section{Acknowledgements}

We wish to express our hearty thanks to Director Culator Yoon Kee Kim, Senior Researcher Seong Jin Kang and Researcher Sei Ho Jung of Cheju-do Folklore and Natural History Museum, for their kind help on our collecting trip on Chejudo Island. Our cordial thanks are due to Prof. Katsura Morimoto and Associate Prof. Osamu Tadauchi of the Entomological Laboratory, Faculty of Agriculture, Kyushu University for his continuous guidance and valuable advices. We are also much indebted to Associate Prof. Michitaka Chûjô of Hikosan Biological Laboratory, Kyushu University for his kid help and advices in various ways for this work. 


\section{REFERENCES}

Cho, P. S., 1957. A systematic catalogue of Korean Coleoptera. Humanities and Sciences, Korea Univ., 2 . 173-338. (In Korean.) 1963. Insects of Querpart Island (Cheju-do). ibid. 6: 159-242. (In Korean.)

Cho, Y. B. \& C. E. Lee, 1986. Phylogenetic relationships among Tribes of Silphidae I (Coleoptera). Nature and Life, 16 (1): 19-25.

- \& 1986b. Notes on Eusilpha jakowlewi (Semenow) and E. japonica (Motschulsky) recorded in Korea (Coleoptera, Silphidae). ibid., 16(2): 57-60.

Harold, E., 1877. Beiträge zur Käferfauna von Japan. Deutsch. ent. Zeitschr.., 21 (2): 337-367.

Hatch, M. H., 1928. Silphidae II. In Junk, W., \& S. Schenkling (eds.), Coleopterorum Catalogus, (95): l-244.

Kim, C. W., 1978. Distribution Atlas of Insects of Korea, Ser. 2, Coleoptera. Korea Univ. Press, Seoul, 414pp.

Kraatz, G., 1877. Japanische Silphidae. Deutsch. ent. Zeitschr.., 21 (1): 100-107.

Kurosawa, Y., 1985. Siiphidae. In Uéno, S.-I., Y. Kurosawa \& M. Satô (eds.), The Coleoptera of Japan in Color, 2: 247-252. (In Japanese.)

Lee, Y. I., W. T. Kim \& D. H. Kim, 1985. Insect fauna of Mt. Halla. In Rep. acad. Surv. Hallasan (Mt.) Nat. Pres.: 351-455. (In Korean.)

Nishikawa, M., 1986. New silphid beetles of the subgenus Calosilpha (Coleoptera, Silphidae). Ent. Pap. pres. Kurosawa, Tokyo :153-158.

Okamoto, H., 1924. The insect fauna of Querpart Island (Saishiu-to). Bull. Agric. Expt. Stat. Gov. -Gen. Chosen, 1(2):47-233.

Paik, W. H. The burying beetles of Korea. Corentomon, Suwon, 1 (1) : 2-12.

Portevin, G., 1926. Les grands Nécrophages du globe, In Encycl, ent., Paris, (A) 6: l-270.

Satô, M. \& S. Saitô, 1989, Coleoptera, Silphidae, In Hirashima, Y. (ed), A Check List of Japanese Insects, Fukuoka, 1:254-256.

Seok, D. M., 1970, The insect fauna of the Is. Querpart. Bojinjae, Seoul,186pp.

Shibata, T., 1969. Some reports on the burying beetles from Japan, I (Col., Silphidae). Ent. Rev. Japan, 21: 47-54. 